\title{
Ethical to using rats in the scientific researches
}

\begin{abstract}
Animal research has had an essential role in various scientific and medical progress along the way from the past century and continuous future to support our understanding of many illnesses. The study gives an outline of using an animal in scientific research. Even though more studies work displayed on Ethical to using rats in the experimental research but there is not enough scientific data or ethic respect to any ethical rules. The data of the present study of the Ethics Committee was collected from internet literature review; a questionnaire was evaluated by researchers from 2004 to 2017 , and review many publications to provide a better understanding of animal research welfare. The survey revealed $10 \%$ of studies involving research animals only use rats in an ethical way and with interest and noble purpose. In conclusions, the present work showed that in spite of the guidelines dictating the use of research animals occurred, there are not enough guarantees and defenses rules. Animal welfare concern in the scientific community needs to precede new vision and mission of journal guidelines, institutions, research funding agencies and other regulations to use the animal of the studies and how to manage strict rules to measure the argent necessary to use animals. It was anticipated laws were needed to protect using animal research by the scientific committee that needs to more than The Three Rs (3Rs: Replacement, Reduction, Refinement) in scientific testing research.
\end{abstract}

Keywords: animal research, animal welfare guidelines, ethical, questionnaire, replacement, reduction, refinement
Volume 6 Issue I - 2018

\author{
Asmaa Fathi Hamouda, \\ 'Department of Biochemistry, University of Alexandria, Egypt \\ ${ }^{2}$ Medical Laboratory Technology Department, Jazan University, \\ Kingdom of Saudi Arabia
}

Correspondence: Asmaa Fathi Hamouda,Assistant Professor in Faculty of Applied Health Sciences, Jazan University, Saudi Arabia, Tel 00966(0)500257869,

Email asmaakingdom I@yahoo.com

Received: December 29, 2017| Published: January 09, 2018

\section{Introduction}

Animals have been used for research by humans from different cultures. The most used species was a rat, followed by mice, notwithstanding, several projects mentioned a broad range of animals, like rabbits, bovines, pigs, snakes, goats, lizard, hamsters, embryonated eggs and other. ${ }^{1-2}$ Their usage provided answers to questions about health and welfare. People enjoy a healthier quality of life because of this progress, and the consequent development of new medicines and treatments. Despite the fact that, the use of animals in scientific and therapeutic research has been a subject of hot discussion for many years, it still needs more consideration. ${ }^{1-4}$ No honest scientist desires to use animals or induce them unnecessary suffering if it can be bypassed, and therefore scientists admit controls on the use of animals in research. More frequently, the bioscience community believes that animals should be used for analysis only within an ethical frame. ${ }^{5-7}$ The Three Rs (3Rs) in connection with science are leading systems for more ethical use of animals in experimentation. They were first reported by W. M. S. Russell and R. L. Burch in 1959. The 3Rs are (3Rs) (Replacement, Reduction, and Refinement). Replacement is methods which avoid or replace the use of animals in research. The reduction is the use of processes that enable researchers to obtain comparable levels of information from fewer animals or to get more information from the same number of animals. Refinement refers to the use of methods that alleviate or minimize potential pain, suffering or discomfort, and enhance animal well-being for the animals used. ${ }^{8-10}$ Good science and experimental design help to decrease the number of animals used in research as they allow scientists to accumulate data using the smallest number of animals needed. Still, good science also means that enough number must be used to enable accurate statistical study and to generate significant results to prevent the recurrence of measures and the resulting need to use more animals. A group of scientist screening potential anticancer drugs applies the so-called hollow-fiber system, in which tumor cells are increased in a tube-like polymer matrix that is inserted into mice. Drugs are then applied, the tubes removed and the number of cells defined. This system has raised the amount of data that can be collected per animal in some investigations and has, therefore, decreased the number of mice used. ${ }^{5-10}$ Although animals cannot yet be replaced entirely, it is essential that researchers maximize reduction and refinement. Sometimes this is achieved relatively quickly by improving animal husbandry and housing, for example, by enriching their environment. These simple measures within the laboratory aim to satisfy the physiological and behavioral needs of the animals and therefore maintain their wellbeing. Another critical factor is refining the experimental procedures themselves, and perfecting the management of pain. ${ }^{5-10}$

\section{Materials and methods}

\section{Study population}

Personal interview protocols produced by the questions answered about the volunteers' (100 researchers) about "Benefits Arguments and reasons" to morally support Rats research from (2004 to 2017). The development and reporting of the questionnaire followed published recommendations. ${ }^{11-15}$ The framework section pronounced "In this survey, animals intends rat in the first level without avoiding other mammals, such as mice, dogs, cats, and others. The goal of this study to the argument and declare that there are good reasons to maintain animal research and the also a reason to avoid using an animal in research such as animal harmful. In this survey, 'harmful' means such information as pain, suffering (disease/injury, boredom, fear, confinement), and early death. ${ }^{13-15}$ The protocol approved by the University ethics committee.

\section{Sample of the survey}

We give you reasons to prove used an animal in research, and 
we request if you agree with that argument. Then we provide some responses to the discussion the motivation to do research and inquiry. All the arguments and answers in this survey are those commonly made in the literature on animal research. When each argument was performed, it was supported by the question "Is this a good enough cause to justify using animals in medical research?" and other. ${ }^{11-15}$

\section{Statistic}

Data is analysis using IBM SPSS software package version 20.0. The significance of the obtained results analyzed at the $5 \%$ level (SPSS version 20.0).

\section{Result and discussion}

Table 1 shows the results of the present study. Animals practiced for research by humans from various farming. The most used species was a rat, followed by mice. However, several projects discussed a broad range of animals, like rabbits, bovines, pigs, snakes, goats, lizard, hamsters, embryonated eggs and other. ${ }^{1-4}$ It has been figured that over 100 million animals are practiced in the world for research each year. ${ }^{11-15}$ The present study from Questionnaire in 2004 to 2017, the researchers, and review scientific documents. In present opinion study, the current work report that only $10 \%$ use rats in an ethical way and with interest and noble purpose. Most of the researchers (90\%) using the rat to experiment without any goal just to have their master or Ph.D. or to have a promotion. Some of the researchers repeat the job from the previous work without any aim or scientific addition with waste and hurt the experimental animal. We reported the $10 \%$ of scientists including me remember the first time using rat for experiments with a passion for doing a real thing, discovering treatment and changing the world and that is a real reason for using rat in scientific experiment. They treated rat in an ethical way. For example, in my study, the rats were very connected to me. When I enter the animal house, rats were very happy; they look appreciation and stand on their upper legs and raise front limbs and heads, they look like young children when their mother comes home.

Table I Results for questionnaire from 100 researchers about "benefits arguments and reasons" to morally support rats research (2004 to 2017)

\begin{tabular}{llll}
\hline Particular & No. & $\%$ & $\mathbf{P}$ \\
\hline $\begin{array}{l}\text { Use rats in an ethical way with } \\
\text { interest and noble purpose. }\end{array}$ & 10 & 10 & $<0.00 I^{*}$ \\
$\begin{array}{l}\text { Using the rat without any goal(or) } \\
\text { to have master or Ph.D. or }\end{array}$ & 90 & 90 & $<0.00 I^{*}$ \\
Promotion & & & \\
\hline
\end{tabular}

\section{*, Statistically significant at $p \leq 0.05$}

To my care, I pat them gently and put the rat in my pocket then I weigh rats, and the rats looked out of my pocket as he watched from a balcony. Then I play with them, the injection game smoothly with studies dose. When any rat had the problem like a skin rash, $10 \%$ of scientists including me provided the treatment and care to the rats until they recover. They used to use the painless method in the researcher on rats (Refinement). They don't waste experimental animals on one research, as they use a few rats to achieve a noble goal (Reduction) for example $10 \%$ of scientists including me used to work in the group each one uses the same rat at a different point to minimize the number of rats and avoid waste the rat. In contrast to some other researchers (90\%), they used to hit unkindly the experimental animals during weighing and injected study and wasted a large number of the animal. My opinion is to consider that the use of animals in research can be ethically and morally justified. And we look forward to the productive discussion in the future with sacrifices (rats) that participate the earth with us. Right now, to change the world as a mission, scientists will try to find methods and new vision which avoid or replace the use of animals in scientific research (Replacement). We should try to do more the three Rs, The Three Rs (3Rs) of science are guiding principles for the more ethical use of animals in testing (3Rs: Replacement, Reduction, Refinement $)^{8-15}$ and more.

\section{Conclusion}

In conclusion, we should try to find methods which avoid or replace the use of animals in research (Replacement). We should try to do the three Rs, The Three Rs (3Rs) (Replacement, Reduction, Refinement) and more. We should include in every research, master, and Ph.D. which use rat more than a student to use all tissue and organ of the same rat with different noble aim. Furthermore, the global scientific community must have a responsibility to make sure that every research community includes the Consultant specializing in the ethics of scientific research in animal ethics as the supervisor in every study. It should be continuously arranged by journals, institutions and research funding agencies requiring researcher compliance before initiating experimental. We need more scientific research ethics, and we need more safeguards to apply them (Figure 1).

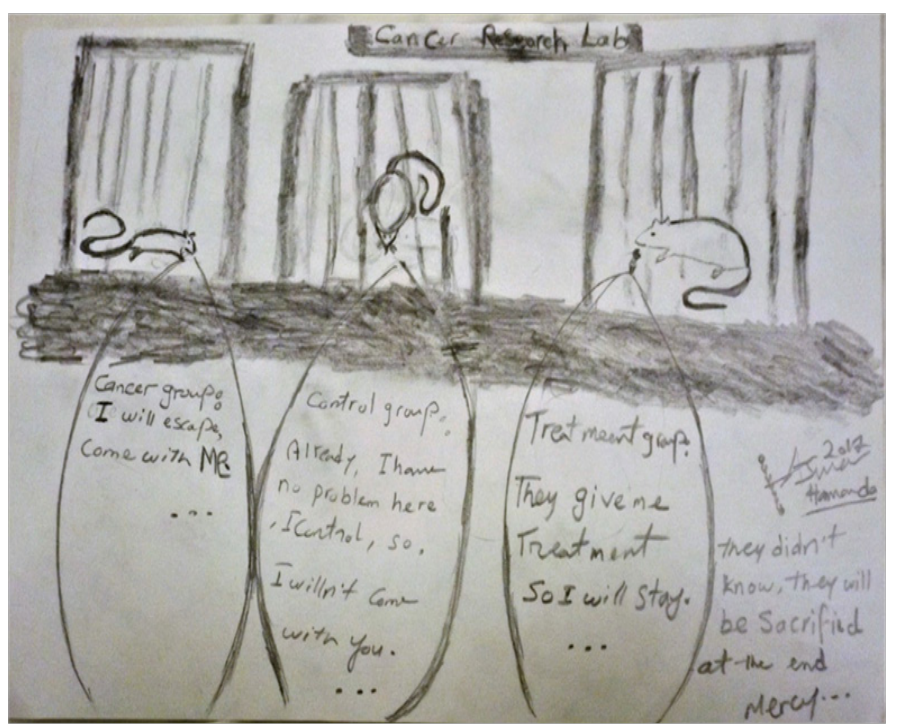

Figure I Now I stop using rats since I am not sure any more about what I achieved before or what I was going to do; I stop trying to change the world anymore where there are other suffer because of me, I mean the rats. I advocate providing new vision in using animals in scientific research. If the animals can speak to me, I will ask them gently for a Consent and Permission to get a biopsy or blood sample, of course, I will do, and we look forward to the productive discussion in the future with sacrifices (rats) that participate the earth with.

\section{Acknowledgements}

The author appreciates Fathi Mousta Hamouda, Taymour-Lank M. Farawilla and People who answer my questionnaire in an honest way.

\section{Conflict of interest}

Author declares that there is no conflict of interest. 


\section{References}

1. Johnston C. Researchers, animals activists fight public relations war at western. CMAJ. 1993;148(8):1349-1353.

2. Ramalli EL, Wanli H, Monica A, et al. A case study from a brazilian medical school and from the international medical literature. Acta Cir Bras. 2012;27(9):659.

3. Srinivasan MR, Sabarinathan A, Geetha A, et al. A Comparative Study on Staining Techniques for Vaginal Exfoliative Cytology of Rat. J Pharmacol Clin Res. 2017;3(3):555615.

4. Conn PM, Parker JV. The animal research war. Faseb J. 2008;22(5):1294-1295.

5. Matthiessen L, Lucaroni B, Sachez E. Towards responsible animal research. EMBO Rep. 2003;4(2):104-107.

6. Double JA. A pharmacological approach for the selection of potential anticancer agents. Altern Lab Anim. 2004;32(Suppl 2):41-48.

7. Schnaider TB. Ethics and research. Acta Cir Bras. 2008;23(1):107-111.

8. Schanaider A, Silva PC. The use of animals in experimental surgery. Acta Cir Bras. 2004;19(4):441-447.
9. Pimenta LG, Silva AL. Ethics in Animal Experimentation. Acta Cir Bras. 2011;16(4):255-260.

10. Russell WMS, Burch RL. The Principles of Humane Experimental Technique. UK: Univ Federation for Animal Welfare; 1959. p. 1-238.

11. Joffe AR, Bara M, Anton N, et al. The ethics of animal research: a survey of pediatric health care workers. Philos Ethics Humanit Med. 2014;9:20.

12. Burns KEA, Duffett M, Kho ME, et al. A guide for the design and conduct of self-administered surveys of clinicians. CMAJ. 2008;179(3):245-252.

13. Ari RJ, Meredith B, Natalie A, et al. The ethics of animal research: a survey of the public and scientists in North America. BMC Med Ethics. 2016;17:17.

14. Knight A. Systematic reviews of animal experiments demonstrate poor contributions to human healthcare. Rev Recent Clin Trials. 2008;3(2):89-96.

15. Paul SM, Mytelka DS, Dunwiddie CT, et al. How to improve R\&D productivity: the pharmaceutical industry's grand challenge. Nat Rev Drug Discov. 2010;9(3):203-214. 